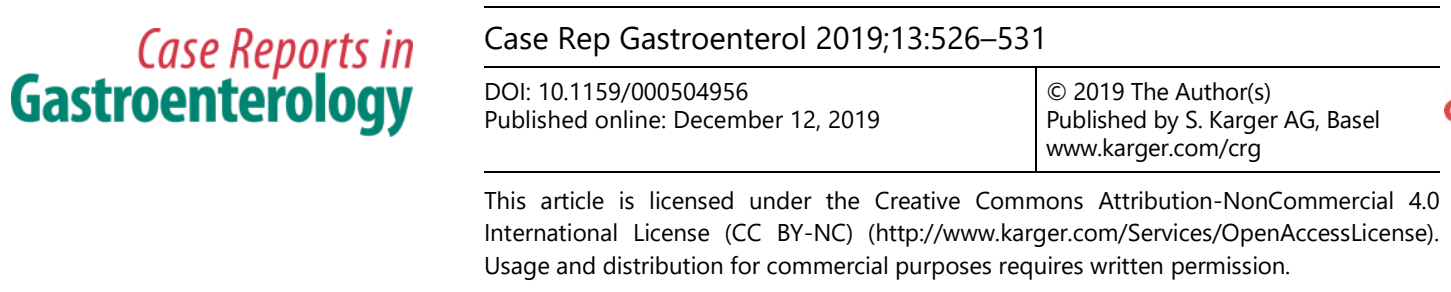

\title{
An Unconventional Diagnostic Method for Cryptosporidial Enteritis in a Healthy Host: Never Call It Quits!
}

\author{
Shivantha Amarnath ${ }^{\mathrm{a}}$ Cheikh Talal El Imad $^{\mathrm{b}}$ Kingsley Ebare $^{\mathrm{c}}$ \\ Hueizhi Wu ${ }^{c}$ Stephen Mulrooney ${ }^{b}$ \\ aDepartment of Internal Medicine, Staten Island University Hospital, Northwell Health, \\ Staten Island, NY, USA; bepartment of Gastroenterology, Staten Island University \\ Hospital, Northwell Health, Staten Island, NY, USA; 'Department of Pathology, Staten \\ Island University Hospital, Northwell Health, Staten Island, NY, USA
}

\section{Keywords}

Cryptosporidium · Endoscopy · Enteritis · Immunocompetent host · Stool culture

\begin{abstract}
Cryptosporidial enteritis has a rising incidence in the USA, mostly affecting immunocompromised individuals and children. It has a self-limiting course in healthy hosts. Herein, we present a unique case of a healthy middle-aged female who presented with a 1-month history of voluminous watery diarrhea and acute blood loss anemia. Cryptosporidial enteritis was diagnosed based on endoscopy with biopsy-proven evidence of 2 jejunal peptic ulcers infected with Cryptosporidium spp. that was originally missed on routine stool culture, ova and parasite tests. The patient was successfully treated with nitazoxanide, and eradication of the protozoan was also confirmed on repeat endoscopic biopsies of the ulcer that were carried out 6 months later. To our knowledge, this is the first case to be reported in the literature with infective colonization of peptic ulcers with Cryptosporidium spp. with consequent systemic symptoms.
\end{abstract}




\section{Case Reports in Gastroenterology}

Case Rep Gastroenterol 2019;13:526-531

DOI: 10.1159/000504956

c) 2019 The Author(s). Published by S. Karger AG, Basel www.karger.com/crg

Amarnath et al.: An Unconventional Diagnostic Method for Cryptosporidial Enteritis in a Healthy Host: Never Call It Quits!

\section{Introduction}

Intestinal cryptosporidiosis is described as a gastroenteritis-like syndrome that is precipitated by the intracellular protozoa, Cryptosporidium spp. The incidence of cryptosporidial enteritis ranges from 10 to $15 \%$ in developing countries and from 1 to $3 \%$ in the USA and Europe [1]. However, the incidence has risen in the USA within the past 10 years due to water-and food-borne outbreaks [2]. For instance, an outbreak of cryptosporidiosis in Wisconsin in 1993 affected over 400,000 people primarily due to a contaminated water supply. This pathogen is known to frequently infect immunocompromised patients but may also cause diarrhea in healthy patients, especially children. In healthy individuals, symptoms are often self-limited. Herein, we describe a unique case of an adult patient with a history of gastric bypass surgery presenting with persistent watery diarrhea and vomiting complicated by microcytic anemia. Initial stool testing, including culture, ova and parasite tests, were unrevealing. Upper endoscopy revealed 2 peptic ulcers at the surgical anastomotic site infected with Cryptosporidium. This is a rare case of intestinal cryptosporidiosis with infective colonization of a peptic ulcer at a surgical site.

\section{Case Report}

A 57-year-old female with a history of gastroesophageal reflux disease, iron deficiency anemia, and a surgical history of Roux-en-Y gastric bypass complicated by jejunal strictures with revision of the gastro-jejunal anastomosis presented with a 7-day history of vomiting and epigastric abdominal pain. The pain was moderate in intensity, intermittent, and crampy. The history dated back to almost 1 month prior, after her return from a 2-week vacation to Latin America. Within 1 week of arrival, she experienced nausea, increased flatus, and progressively worsening voluminous watery diarrhea with 6-7 bowel movements per day associated with intermittent melanotic stools. Her diarrhea slowly subsided to 1-2 bowel movements per day, and she subsequently developed frequent episodes of nonbilious and nonbloody vomiting associated with reduced oral intake. She had lost around 5 pounds from a baseline weight of 161 pounds in the preceding month. She denied any history of fever, chills, allergies, use of laxatives, recent sick contacts, or sexual activity.

Her vital signs were within normal limits except for orthostatic hypotension. Physical exam demonstrated dry oral mucosa with pale conjunctiva. Abdominal exam revealed hyperactive bowel sounds with tenderness to deep palpation of the epigastrium. There was no evidence of rigidity or guarding. There was no evidence of lymphadenopathy, icterus, or rashes. Digital rectal exam uncovered green stool. Laboratory findings were notable for hemoglobin of $9.1 \mathrm{~g} / \mathrm{dL}$, which had dropped from her baseline of $11.2 \mathrm{~g} / \mathrm{dL}$, and hyponatremia of 130 $\mathrm{mmol} / \mathrm{L}$. Hepatic function panel along with serum lipase and lactate were within normal ranges. Computed tomography scan of the abdomen and pelvis with oral and intravenous (IV) contrast showed inflammation around the gastro-jejunostomy site. Blood cultures along with stool studies, including culture, ova, parasites, C. difficile toxin, Giardia antigen, and rotavirus antigen, were unremarkable. The patient was admitted with a working diagnosis of viral gastroenteritis. She was started on anti-emetics, IV pantoprazole, and IV hydration. The patient underwent an upper endoscopy and was found to have 2 ulcers, 1- and 2-cm clean-based ulcers with a deep crater at the anastomotic site (Fig. 1). No active bleeding or any visible vessel was evident. Biopsies of the gastro-jejunostomy anastomotic site ulcers were obtained and demonstrated moderate chronic active inflammation with ulcer formation on certain 


\section{Case Reports in Gastroenterology}

Case Rep Gastroenterol 2019;13:526-531

DOI: 10.1159/000504956

c) 2019 The Author(s). Published by S. Karger AG, Basel www.karger.com/crg

Amarnath et al.: An Unconventional Diagnostic Method for Cryptosporidial Enteritis in a Healthy Host: Never Call It Quits!

fragments along with tissue necrosis (Fig. 2). There were small spherical-shaped bluish-purple organisms, 2-5 $\mu \mathrm{m}$ in diameter, appearing like "blue beads" on hematoxylin and eosin stain. These organisms were mostly seen in the necrotic tissue and were further highlighted by Giemsa and Gram stains. The organisms were also identified by Gomori's methenamine silver nitrate and Masson's trichrome stains. The histopathologic pattern and special stain profile of the organism were suggestive of Cryptosporidium, and a diagnosis of intestinal cryptosporidiosis was made. Biopsies of the healthy tissue surrounding the ulcer sites did not demonstrate any evidence of Cryptosporidium. The patient tested negative for HIV, and laboratory testing for immunodeficiency, including serum immunoglobulin levels, were unremarkable. The patient was started on nitazoxanide $500 \mathrm{mg}$ every $12 \mathrm{~h}$ for 3 days. Her symptoms steadily improved within 3 days with toleration of an oral diet, and she was subsequently discharged home on pantoprazole. The patient underwent a repeat endoscopy 6 months later, and repeat biopsies of the prior ulcers confirmed eradication of the protozoan.

\section{Discussion}

The first human case of cryptosporidial enteritis was described in the literature in 1976 [3]. Interestingly, $6 \%$ of enteritis cases affecting immunocompetent hosts are attributed to Cryptosporidium [4]. The most common human pathogens include C. hominis, C. parvum, $C$. meleagridis, C. felis, C. canis, and C. rabbit genotype [5]. However, the majority of cases of intestinal cryptosporidiosis are caused by C. parvum and $C$. hominis [1].

Children, international travelers, farmers, and those who have access to only poor-quality drinking water are the most frequent targets of intestinal cryptosporidiosis. Transmission is fecal-oral via contaminated food, water, and fomites and is acquired via ingestion of oocysts. Daycare centers and swimming pools are the most common locations harboring this infection in developed countries [6]. Cryptosporidial enteritis in healthy hosts is often a self-limiting disease but can be life-threatening in immunocompromised patients, namely organ transplant recipients and HIV patients with CD4 count $<100$, and in severe combined immune deficiency as well as hyper-IgM syndrome [5]. Patients with inflammatory bowel disease receiving steroids or biological treatment are at increased risk of infection from Cryptosporidium. Intriguingly, recent studies have shown that Cryptosporidium-triggered enteritis occurred more frequently in patients with colorectal cancer, even before therapy had commenced, and was independent of age and sex [1].

The primary site for colonization is the small intestine (jejunum and ileum) in both immunocompetent and immunocompromised hosts; however; it can involve the colon, biliary tract, and pancreas in the latter population [5]. Symptoms may include abdominal pain, diarrhea, vomiting, weight loss, abdominal bloating, and fever. However, if it involves the biliary tree, cryptosporidiosis can present with a clinical picture of pancreatitis, sclerosing cholangitis, and even liver cirrhosis. Diarrhea can be osmotic, inflammatory, or secretory with 3-6 bowel movements per day, although the causative mechanism has not been fully established thus far. It has been proposed that the pathogen may damage the epithelial layer lining the intestine and biliary tract impairing absorption and promoting secretion. It is often difficult to distinguish an inflammatory bowel disease flare from cryptosporidial enteritis, and cryptosporidial enteritis should be considered as a possible etiology in patients presenting with massive stool output. Symptoms can last up to 90-100 days even in immunocompetent patients based on the data from case studies published in the UK and Australia [5]. The oocysts can continue to shed up 2 months even after symptoms have ceased. 


\section{Case Reports in Gastroenterology}

Case Rep Gastroenterol 2019;13:526-531

DOI: $10.1159 / 000504956$

(c) 2019 The Author(s). Published by S. Karger AG, Basel www.karger.com/crg

Amarnath et al.: An Unconventional Diagnostic Method for Cryptosporidial Enteritis in a Healthy Host: Never Call It Quits!

Diagnosis of cryptosporidial enteritis is often missed on routine stool culture, ova and parasite tests due to the modified acid-fast stain (modified Ziehl-Neelsen or auramine) having a sensitivity of only $50 \%$ compared to enzyme immunoassay and direct fluorescent antibody methods that carry a sensitivity and specificity of $>90 \%$ [7]. This holds true for our patient as her initial stool culture, ova and parasite tests did not uncover any Cryptosporidium spp. The convenient dot blot hybridization assay carries a sensitivity of $95 \%$ and a specificity of $99.7 \%$ [8]. A study proposed that a triple feces test approach, obtaining stool samples on 3 consecutive days and fixating and concentrating the samples before staining, can increase its detection rate [5]. Our case also highlights the fact that in patients with a high index of suspicion of cryptosporidial enteritis and co-existing complications, such as anemia, endoscopic biopsies of the small intestine can aid in the diagnosis and isolation of the pathogen.

Nitazoxanide, a thiazolide with broad-spectrum activity against enteric protozoans, is FDA approved for the treatment of cryptosporidiosis in nonimmunocompromised adults and children as it can reduce the duration of symptoms, locust shedding, and elimination of the pathogen, which has been demonstrated in several placebo-controlled trials $[9,10]$. The recommended dose is $500 \mathrm{mg}$ every $12 \mathrm{~h}$ for adults for a total of 3 days [6]. Prevention is key, ergo proper hand washing when cleaning toilets, changing diapers, and caring for those with diarrhea, and proper disposal of excrements is important. Patients with intestinal cryptosporidiosis should also be advised that they should avoid using swimming pools for 2 weeks [5]. The key factor in immunity and clearance of the parasite is primarily dependent on CD4 T cells. Hence, highly active antiretroviral therapy (HAART) is the most effective treatment for reducing the severity and frequency of cryptosporidiosis in AIDS patients [9].

Cryptosporidial enteritis is a significant cause of persistent diarrhea in both healthy and immune-compromised individuals and should be considered as a key differential diagnosis especially in those presenting with persistent voluminous diarrhea. Its incidence has risen within the past several years, and novel diagnostic modalities have proven useful in identifying this protozoan in stool samples. Negative stool culture, ova and parasite tests do not rule out the possibility of Cryptosporidium as a cause of chronic diarrhea in immunocompetent patients. Hence, utilization of the triple feces test approach or even endoscopic biopsies in highrisk patients may raise the sensitivity of diagnosing cryptosporidial enteritis. Expectant management is a plausible option for most immunocompetent patients, and the use of nitazoxanide should be considered especially in those presenting with severe acute symptoms or symptoms lasting more than 14 days. Further studies need to be carried out to determine if any optimal tests can be used to confirm eradication of this protozoan.

\section{Acknowledgements}

We would like to thank the patient for allowing us to share the details of her clinical history. We would also like to express our appreciation to the Pathology Department at Staten Island University Hospital for providing us with the pathology slides.

\section{Statement of Ethics}

We hereby confirm that informed consent was obtained from the patient for publication of the case details. 


\section{Case Reports in Gastroenterology}

Amarnath et al.: An Unconventional Diagnostic Method for Cryptosporidial Enteritis in a Healthy Host: Never Call It Quits!

\section{Disclosure Statement}

The authors have no conflicts of interest to declare.

\section{Funding Sources}

There are no financial disclosures or funding.

\section{Author Contributions}

Shivantha Amarnath, MD: literature research, writing the manuscript. Chiekh Talal El Imad, MD: editing and reviewing the manuscript. Kingsley Ebare, MD: contribution with pathological slides and relative content. Hueizhi Wu, MD: study mentor for the pathology slides and critical revision of the pathology slides content. Stephen Mulrooney, MD: study mentor, critical revision of the manuscript.

\section{References}

1 Sulżyc-Bielicka V, Kołodziejczyk L, Jaczewska S, Bielicki D, Safranow K, Bielicki P, et al. Colorectal cancer and Cryptosporidium spp. infection. PLoS One. 2018 Apr;13(4):e0195834.

2 Painter JE, Hlavsa MC, Collier SA, Xiao L, Yoder JS; Centers for Disease Control and Prevention. Cryptosporidiosis surveillance-United States, 2011-2012. MMWR Suppl. 2015 May;64(3):1-14.

3 Ogata S, Suganuma T, Okada C, Inoue K, Kinoshita A, Sato K. A case of sporadic intestinal cryptosporidiosis diagnosed by endoscopic biopsy. Acta Med Okayama. 2009 Oct;63(5):287-91.

4 Mainali NR, Quinlan P, Ukaigwe A, Amirishetty S. Cryptosporidial diarrhea in an immunocompetent adult: role of nitazoxanide. J Community Hosp Intern Med Perspect. 2013 Dec;3(3-4):21075.

5 Chalmers RM, Davies AP. Minireview: clinical cryptosporidiosis. Exp Parasitol. 2010 Jan;124(1):138-46.

6 Kenny JM, Kelly P. Protozoal gastrointestinal infections. Medicine (Baltimore). 2009 Nov;37(11):599-602.

7 Cheung DA, Langshaw A, Rivera-Rivera E. Cryptosporidium diagnosed on endoscopic biopsy in a paediatric patient with inflammatory bowel disease. BMJ Case Rep. 2018 Jan;2018. https://doi.org/10.1136/bcr-2017222015.

8 Khalil S, Mirdha BR, Paul J, Panda A, Makharia G, Chaudhry R, et al. Development and evaluation of molecular methods for detection of Cryptosporidium spp. in human clinical samples. Exp Parasitol. 2016 Nov;170:20713.

9 Rossignol JF. Cryptosporidium and Giardia: treatment options and prospects for new drugs. Exp Parasitol. 2010 Jan;124(1):45-53.

10 Le TT, Bilal M, Reep G. Yellow Colon: A Case of Cryptosporidium Colitis. Clin Gastroenterol Hepatol. 2017 Jun;15(6):A21-2. 


\section{Case Reports in Gastroenterology}

\begin{tabular}{l|l}
\hline Case Rep Gastroenterol 2019;13:526-531 \\
\hline DOI: 10.1159/000504956 & $\begin{array}{l}\text { @ 2019 The Author(s). Published by S. Karger AG, Basel } \\
\text { www.karger.com/crg }\end{array}$ \\
\hline
\end{tabular}

Amarnath et al.: An Unconventional Diagnostic Method for Cryptosporidial Enteritis in a Healthy Host: Never Call It Quits!
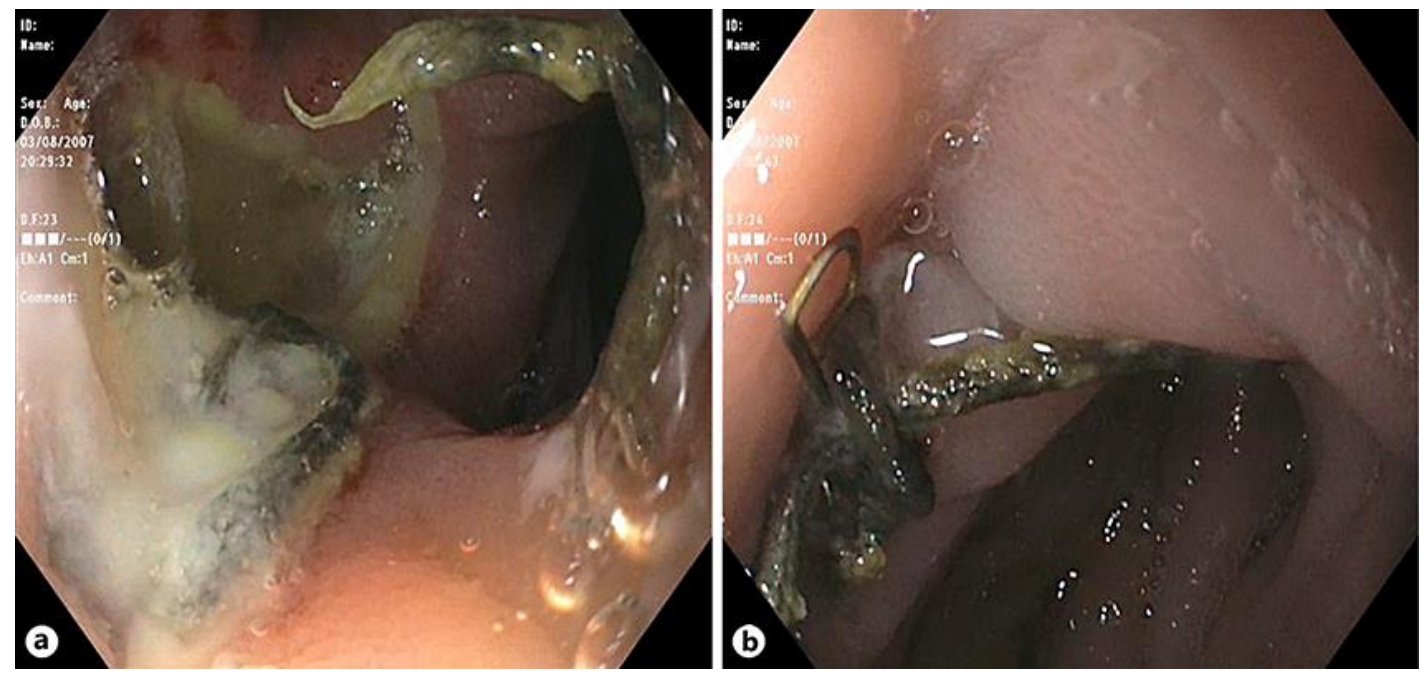

Fig. 1. a, b Endoscopic view of the peptic ulcers at the gastro-jejunostomy anastomotic site.

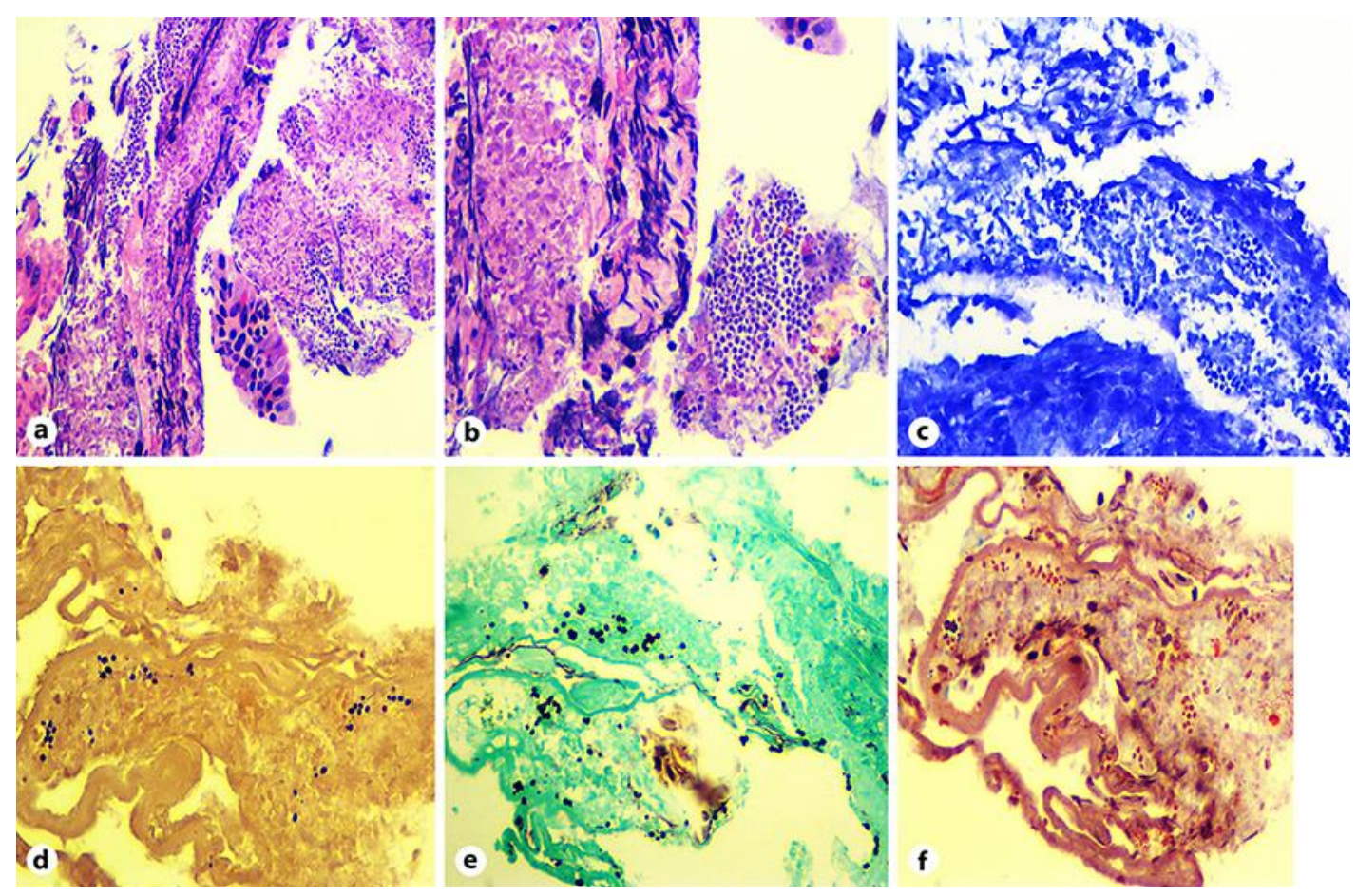

Fig. 2. Cryptosporidium at the necrotic tissue of the anastomotic site. a HE, $\times 40$. Cryptosporidium appearing as small bluish-purple spherical organisms. b HE, $\times 60$. c Giemsa stain, $\times 60$. $\mathbf{d}$ Gram stain, $\times 60$. e Gomori's methenamine silver nitrate stain, $\times 60$. $f$ Trichrome stain, $\times 60$. Cryptosporidium appearing as small dark pink to orange spherical organisms. 